\title{
Edge-Transitive Cyclic Covers of Complete Graphs with Prime Power Order
}

\author{
Zhaohong Huang1, Yin Liu \\ ${ }^{1}$ School of Mathematics and Statistics Science, Ludong University, Yantai, China \\ ${ }^{2}$ School of Mathematics and Statistics, Yunnan Normal University, Kunming, China \\ Email: huangzhaohong1987@163.com, liuyinjiayou@sina.com
}

How to cite this paper: Huang, Z.H. and Liu, Y. (2022) Edge-Transitive Cyclic Covers of Complete Graphs with Prime Power Order. Journal of Applied Mathematics and Physics, 10, 289-300.

https://doi.org/10.4236/jamp.2022.102022

Received: January 6, 2022

Accepted: February 11, 2022

Published: February 14, 2022

Copyright () 2022 by author(s) and Scientific Research Publishing Inc. This work is licensed under the Creative Commons Attribution International License (CC BY 4.0).

http://creativecommons.org/licenses/by/4.0/

\begin{abstract}
Characterizing regular covers of symmetric graphs is one of the fundamental topics in the field of algebraic graph theory, and is often a key step for approaching general symmetric graphs. Complete graphs, which are typical symmetric graphs, naturally appear in the study of many symmetric graphs as normal quotient graphs. In this paper, a characterization of edge-transitive cyclic covers of complete graphs with prime power order is given by using the techniques of finite group theory and the related properties of coset graphs. Certain previous results are generalized and some new families of examples are founded.
\end{abstract}

\section{Keywords}

Cover, Complete Graph, Normal Quotient Graph, Automorphism

\section{Introduction}

Throughout the paper, by a graph, we mean a connected, undirected and simple graph with a valency of at least three.

For a graph $\Gamma$, denote its vertex set, edge set and arc set by $V \Gamma, E \Gamma$ and $A \Gamma$ respectively. Then, for an automorphism group $X \leq A u t \Gamma, \Gamma$ is called $X$-vertex-transitive, $X$-edge-transitive or $X$-arc-transitive, if $X$ is transitive on $V \Gamma, E \Gamma$ or $A \Gamma$, respectively; $\Gamma$ is called $X$-locally-primitive if the vertex stabilizer $X_{\alpha}:=\left\{x \in X \mid \alpha^{x}=\alpha\right\}$ acts primitively on the neighbor set $\Gamma(\alpha)$ for each $\alpha \in V \Gamma$; and $\Gamma$ is called $(X, 2)$-arc-transitive if $X$ is transitive on the set of 2 -arcs (that is, the sequences of three distinct adjacent vertices) of $\Gamma$. It is known that a locally-primitive graph is edge-transitive, and a 2 -arc-transitive graph is locally-primitive.

Let $\Gamma$ and $\Sigma$ be two graphs. Then $\Gamma$ is called a cover (or covering) of $\Sigma$ 
with a projection $\rho$, if $\rho$ is a surjection from $V \Gamma$ to $V \Sigma$ such that the restriction $\left.\rho\right|_{\Gamma(\tilde{\alpha})}: \Gamma(\tilde{\alpha}) \rightarrow \Sigma(\alpha)$ is a bijection for each $\alpha \in V \Sigma$ and preimage $\tilde{\alpha} \in V \Gamma$ of $\alpha$ under $\rho$. Further, $\Gamma$ is called a $K$-cover (or regular $K$-cover) if there is a semiregular subgroup $K \leq$ Aut $\Gamma$ such that $\Sigma$ is isomorphic to the quotient graph $\Gamma_{K}$, say by $\phi$, and the quotient map $\Gamma \rightarrow \Gamma_{K}$ is the composition $\rho \phi$. For each vertex $\alpha \in V \Sigma$, the set of all preimages of $\alpha$ under $\rho$ is called a fibre. An automorphism of $\Gamma$ is called fibre-preserving if it maps each fibre to a fibre. The group, consisting of all fibre-preserving automorphisms of $\Gamma$, is called the fibre-preserving group. Then the fibre set forms an invariant partition of the fibre-preserving group on $V \Gamma$, and the fibre-preserving group has a natural induced action on the fibre set. The kernel of this action is called the covering transformation group.

A typical method for studying transitive graphs is taking normal quotient graphs. It is well known that each edge-transitive graph is a cover or multi-cover of a "basic" graph: a vertex quasiprimitive or vertex biquasiprimitive graph, and in particular, each vertex-transitive locally-primitive graph is a cover of a basic graph (see [1]). This suggests a two-step strategy for characterizing transitive graphs: step 1 is to obtain basic graphs, and step 2 is to find covers of the obtained basic graphs.

Therefore, characterizing transitive covers of given graphs is often a key step for approaching general transitive graphs. Numerous relative results have been obtained in the literature, see [2] [3] [4] [5] and reference therein for edge- or arc-transitive cyclic or abelian covers of graphs with small order. As typical symmetric graphs, complete graphs often naturally appear (as normal quotient graphs) in the studying of many families of graphs, characterize their covers has been receiving much attention. For example, [4] classifies arc-transitive cyclic and elementary abelian covers of $\mathrm{K}_{4}$, [2] classifies arc-transitive abelian covers of $\mathrm{K}_{4}$, and [6] classifies arc-transitive cyclic covers of $\mathrm{K}_{5}, \mathrm{~K}_{6}$ and $\mathrm{K}_{7}$. In particular, 2-arc-transitive cyclic, $\mathbb{Z}_{p}^{2}$ - and $\mathbb{Z}_{p}^{3}$-covers with $p$ a prime of complete graphs are determined in [7] [8]. It is a next interesting topic to characterize covers of complete graphs with a "weak" symmetry. The main purpose of this paper is to characterize edge-transitive cyclic covers of complete graphs with prime power order.

The terminologies and notations used in this paper are standard. For example, for a positive integer $n$, denote by $\mathbb{Z}_{n}$ the cyclic group of order $n$; given two groups $N$ and $H$, denote by $N \times H$ the direct product of $N$ and $H$, by $N . H$ an extension of $N$ by $H$, and if such an extension is split, then we write $N: H$ instead of N.H; also, a group $G$ is called a central product of two subgroups $S$ and $T$, denoted by $G=S \circ T$, if $G=S T$, the commutator subgroup $[S, T]=1$, and $S \cap T$ is contained in the center of $G$, see ([9]: p. 141).

Theorem 1.1. Let $\Gamma$ be an edge-transitive $\mathbb{Z}_{m}$-cover of the complete graph $\Sigma \cong \mathrm{K}_{p^{n}}$ with p a prime. Then one of the following holds.

1) $\Gamma$ is 2-arc-transitive, and either 
i) $m=2$ and $\Gamma \cong \mathrm{K}_{p^{n}, p^{n}}-p^{n} \mathrm{~K}_{2} ;$ or

ii) $m=4, \quad \Sigma \cong \mathrm{K}_{2^{n}}$ with $2^{n}-1 \geq 7$ a prime, and $\Gamma \cong X_{1}\left(4,2^{n}-1\right)$, as in Example 3.4.

2) $\Sigma \cong \mathrm{K}_{2^{n}}$ with $2^{n}-1 \geq 7$ a prime, and either

i) $m \mid 2^{n}-2$ and $\Gamma \cong \mathcal{G}^{\{1\}}$ (as in Example 3.5) is $\mathbb{Z}_{m} \times \operatorname{PSL}\left(2,2^{n}-1\right)$-locallyprimitive, or

ii) $m \mid 2^{n+1}-4$ is even and $\Gamma \cong \mathcal{G}^{\{2\}}$ (as in Example 3.7) is $\mathbb{Z}_{m} \circ \operatorname{PSL}\left(2,2^{n}-1\right)$-locally-primitive.

3) $\Gamma$ is $\mathbb{Z}_{m} \times \operatorname{PSL}(d, q)$-arc-transitive with vertex stabilizer isomorphic to a maximal parabolic subgroup of $\operatorname{PSL}(d, q)$, where $m \mid 2(d-1, q-1), d \geq 3$ is a prime and $d \nmid q-1$ such that $\frac{q^{d}-1}{q-1}=p^{n}$.

4) $\Gamma$ is a normal edge-transitive Cayley graph.

For given $m, d, q$, graphs in part (3) may be determined (for example, a simple way is using computer software MAGMA or GAP), and graphs in part (4) have a nice construction and can be well understood (see [10]: Section 2). However, it seems hard to give a specific classification of these graphs.

In locally-primitive cyclic cover case, we have the following nice version.

Theorem 1.2. Let $\Gamma$ be a locally-primitive $\mathbb{Z}_{m}$-cover of the complete graph $\Sigma=\mathrm{K}_{p^{n}}$ with $p$ a prime. Then one of the following is true.

1) $\Gamma$ is 2-arc-transitive, and either

i) $m=2$ and $\Gamma \cong \mathrm{K}_{p^{n}, p^{n}}-p^{n} \mathrm{~K}_{2} ;$ or

ii) $m=4, \quad \Sigma \cong \mathrm{K}_{2^{n}}$ with $2^{n}-1 \geq 7$ a prime, and $\Gamma \cong X_{1}\left(4,2^{n}-1\right)$.

2) $\Sigma \cong K_{2^{n}}$, and one of the following holds.

i) $2^{n}-1 \geq 7$ is a prime, $m \mid 2^{n}-2$, and $\Gamma \cong \mathcal{G}^{\{1\}}$.

ii) $2^{n}-1 \geq 7$ is a prime, $m \mid 2^{n+1}-4$, and $\Gamma \cong \mathcal{G}^{\{2\}}$.

iii) $m=2^{d}$, and $\Gamma$ is a normal locally-primitive Cayley graph of a 2-group isomorphic to $\mathbb{Z}_{2^{d}} \cdot \mathbb{Z}_{2}^{n}$.

Theorem 1.2 has the following interesting consequences.

Corollary 1.3. A locally-primitive cyclic cover of a complete graph $\mathrm{K}_{p^{n}}$ with $p$ an odd prime is isomorphic to $\mathrm{K}_{p^{n}, p^{n}}-p^{n} \mathrm{~K}_{2}$.

Corollary 1.4. Let $\Gamma$ be a locally-primitive $\mathbb{Z}_{m}$-cover of the complete graph $\mathrm{K}_{p^{2}}$ with $p$ a prime. Then one of the following holds.

1) $m=2$ and $\Gamma=\mathrm{K}_{p^{2}, p^{2}}-p^{2} \mathrm{~K}_{2}$;

2) $p=2, \quad m=4$ and $\Gamma=P(8,3)$ is the generalized Peterson graph.

This paper is organized as follows. After this introduction, some preliminary results are given in Section 2. By giving some technical lemmas and introducing examples appearing in Theorem 1.1 in Section 3, we present the proofs of Theorems 1.1 and 1.2 in Section 4.

\section{Preliminaries}

The following proposition classifies nonabelian simple groups with a subgroup 
of prime power index.

Proposition 2.1. ([11]) Let $T$ be a nonabelian simple group which has a subgroup $H$ with index a prime power $p^{n}$. Then the tuple $(T, H)$ satisfies one of the following.

1) $T \cong \mathrm{A}_{p^{n}}$, and $H \cong \mathrm{A}_{p^{n-1}}$;

2) $T \cong \operatorname{PSL}(d, q)$, and $H$ is a maximal parabolic subgroup of $T$ such that $\frac{q^{d}-1}{q-1}=p^{n}$;

3) $T \cong \mathrm{PSL}_{2}(11)$ and $H \cong \mathrm{A}_{5}$;

4) $T \cong \mathrm{M}_{11}$ and $H \cong \mathrm{M}_{10}$;

5) $T \cong \mathrm{M}_{23}$ and $\mathrm{H} \cong \mathrm{M}_{22}$;

6) $T \cong \operatorname{PSU}(4,2)$ and $H \cong \mathbb{Z}_{2}^{4}: \mathrm{A}_{5}$.

Let $a$ and $d$ be positive integers. A prime $r$ is called a primitive prime divisor of $a^{d}-1$ if $r$ divides $a^{d}-1$ but does not divide $a^{i}-1$ for all $1 \leq i \leq d$. The following is a well-known result of Zsigmondy.

Theorem 2.2. ([12]: p. 508) For any positive integers $a$ and $d$, either $a^{d}-1$ has a primitive prime divisor, or $(d, a)=(6,2)$ or $\left(2,2^{m}-1\right)$, where $m \geq 2$.

A graph $\Gamma$ is called a Cayley graph of a group $G$ if there is a subset $S \subseteq G \backslash\{1\}$, with $S=S^{-1}:=\left\{g^{-1} \mid g \in S\right\}$, such that $V \Gamma=G$, and two vertices $g$ and $h$ are adjacent if and only if $h g^{-1} \in S$. This Cayley graph is denoted by Cay $(G, S)$. In particular, if $\Gamma=\operatorname{Cay}(G, S)$ is $X$-edge-transitive or $X$-locally-primitive and the right regular representation $\hat{G} \triangleleft X \leq$ Aut $\Gamma$, then $\Gamma$ is called $X$-normal edge-transitive or $X$-normal locally-primitive Cayley graph respectively. Normal edge-transitive Cayley graphs have some nice properties, see [10].

Lemma 2.3. Let $\Gamma=\operatorname{Cay}(G, S)$ be an $X$-normal locally-primitive Cayley graph of an abelian group $G$. Then $G$ is an elementary abelian 2-group.

For an $X$-vertex-transitive graph $\Gamma$ with $X \leq$ Aut $\Gamma$, if $X$ has an intransitive normal subgroup $N$, denote by $V \Gamma_{N}$ the set of all $N$-orbits on $V \Gamma$. The normal quotient graph of $\Gamma$ induced by $N$, denoted by $\Gamma_{N}$, is defined with vertex set $V \Gamma_{N}$ and two vertices $B, C \in V \Gamma_{N}$ are adjacent if and only if some vertex in $B$ is adjacent in $\Gamma$ to some vertex in $C$. In particular, if for each adjacent vertices $B, C \in V \Gamma_{N}$, the induced subgraph $[B, C] \cong k \mathrm{~K}_{2}$ where $k=|B|=|C|$, then $\Gamma$ is a $N$-cover of $\Gamma_{N}$.

The following theorem plays an important role for the studying of locally-primitive graphs, which is first proved by Praeger ([1]: Theorem 4.1) for 2-arc-transitive case and slightly generalized to locally-primitive case by ([13]: Lemma 2.5).

Theorem 2.4. Let $\Gamma$ be an $X$-vertex-transitive locally-primitive graph, and let $N \triangleleft X$ have at least three orbits on $V \Gamma$. Then the following statements hold.

1) $N$ is semiregular on $V \Gamma, X / N \leq$ Aut $\Gamma_{N}, \Gamma_{N}$ is X/N-locally-primitive, and $\Gamma$ is a $N$-cover of $\Gamma_{N}$;

2) $\Gamma$ is $(X, s)$-arc-transitive if and only if $\Gamma_{N}$ is $(X / N, s)$-arc-transitive, where $1 \leq s \leq 5$ or $s=7$; 
3) $X_{\alpha} \cong(X / N)_{\delta}$, where $\alpha \in V \Gamma$ and $\delta \in V \Gamma_{N}$.

Let $G$ be a group, $H$ a core-free subgroup of $G$ (that is, $H$ contains no nontrivial normal subgroup of $G$ ), and $g \in G \backslash H$. The coset digraph $\operatorname{Cos}(G, H, H g H)$ is defined with vertex set $[G: H]$ such that

$$
H x \sim H y \Leftrightarrow y x^{-1} \in H g H .
$$

The following properties of coset digraphs are known, refer to [14] or [15].

Lemma 2.5. Using notations as above, and let $\Gamma=\operatorname{Cos}(G, H, H g H)$. Then

1) $\Gamma$ is undirected if and only if $g^{2} \in H$. In this case, $\Gamma$ is $G$-arc-transitive with valency $\left|H: H \cap H^{g}\right|$;

2) $\Gamma$ is connected if and only if $\langle H, g\rangle=G$;

Conversely, each $G$-arc-transitive graph is isomorphic to $\operatorname{Cos}\left(G, G_{\alpha}, G_{\alpha} f G_{\alpha}\right)$, where $f$ is a 2 -element of $G$ such that $f^{2} \in G_{\alpha}$ and $\left\langle G_{\alpha}, f\right\rangle=G$.

Let $G=N . H$ be an extension. If $N$ is in the center of $G$, then the extension is called a central extension. A group $G$ is said to be perfect if $G=G^{\prime}$, the commutator subgroup of $G$. For a given group $H$, if $N$ is the largest abelian group such that $G:=N . H$ is perfect and the extension is a central extension, then $N$ is called the Schur Multiplier of $H$, denoted by $\operatorname{Mult}(H)$. The following lemma is known.

Lemma 2.6. Assume that $G=N . T$, where $N$ is a cyclic group and $T$ is a nonabelian simple group. Then $G=N . T$ is a central extension. Further, $G=N G^{\prime}$ and $G^{\prime}=M . T$ is a perfect group, where $M \leq N \cap \operatorname{Mult}(H)$.

The Schur multipliers of nonabelian simple groups are known, refer to ([16]: pp. 302-303).

Lemma 2.7. Let $T=\operatorname{PSL}(d, q)$. Then $\operatorname{Mult}(T) \cong \mathbb{Z}_{(d, q-1)}$ with the only exceptions in the following Table.

\begin{tabular}{cccccc}
\hline$(d, q)$ & $(2,4)$ & $(2,9)$ & $(3,2)$ & $(3,4)$ & $(4,2)$ \\
\hline $\operatorname{Mult}(T)$ & $\mathbb{Z}_{2}$ & $\mathbb{Z}_{2} \times \mathbb{Z}_{3}$ & $\mathbb{Z}_{2}$ & $\mathbb{Z}_{3} \times \mathbb{Z}_{4}^{2}$ & $\mathbb{Z}_{2}$ \\
\hline
\end{tabular}

\section{Technical Lemmas and Example}

In this section, we prove certain technical lemmas and introduce examples appearing in Theorem 1.1.

\subsection{Lemmas}

Lemma 3.1. Suppose $\frac{q^{d}-1}{q-1}=p^{n}$, where $q$ is a prime power and $p$ is a prime. Then $d$ is a prime. In particular, if $q=2$, then $n=1$.

Proof. Suppose on the contrary, $d$ is not a prime. Let $r$ be a prime divisor of $d$. Since $2^{6}-1=63$ is not a prime power, $(d, q) \neq(6,2)$. It then follows from Theorem 2.2 that $q^{d}-1$ has a primitive prime divisor, say $t$. Thus $t$ divides $\frac{q^{d}-1}{q-1}=p^{n}$, implying $t=p$. However, as $\frac{q^{r}-1}{q-1}$ divides $\frac{q^{d}-1}{q-1}$, we have 
$\frac{q^{r}-1}{q-1}$ is also a power of $p$, and hence $t=p$ divides $q^{r}-1$, which is a contradiction.

Now, suppose $q=2$. Then $p$ is odd. If $n$ is even, then $2^{d}=p^{n}+1 \equiv 2(\bmod 4)$, yielding a contradiction. Thus $n$ is odd. Since $p+1 \mid p^{n}+1, \quad p+1=2^{s}$ for some $2 \leq s \leq d$, so $2^{s}-1=p$ divides $p^{n}=2^{d}-1$. It follows $2^{s}-1=\left(2^{s}-1,2^{d}-1\right)=2^{(s, d)}-1$, implying $s=d$ as $s \geq 2$ and $d$ is a prime. Hence $p=2^{s}-1=2^{d}-1=p^{n}$ and $n=1$.

Lemma 3.2. Suppose $G=N H \leq \operatorname{Sym}(\Omega)$, where $N \triangleleft G$ and $H \leq G$. Then $G_{\alpha}=N_{\alpha} . o$, where $\alpha \in \Omega$ and $o \leq H /(H \cap N)$. In particular, if $N$ is transitive on $\Omega$, then $o \cong H /(H \cap N)$.

Proof. Since $N \triangleleft G, N_{\alpha} \triangleleft G_{\alpha}$. As

$G_{\alpha} / N_{\alpha}=G_{\alpha} /\left(N \cap G_{\alpha}\right) \cong N G_{\alpha} / N \leq G / N=N H / N \cong H /(H \cap N)$, we have

$o:=G_{\alpha} / N_{\alpha} \leq H /(H \cap N)$. Particularly, if $N$ is transitive on $\Omega$, then $N G_{\alpha}=G$ and hence $o \cong H /(N \cap H)$.

The next lemma is possibly known. As the authors find no proper references, a proof is given.

Lemma 3.3. Let $\Gamma$ be an $X$-vertex-transitive $K$-cover of $\Sigma$ with $X \leq$ Aut $\Gamma$. Then $X_{\alpha} \cong(X / K)_{\delta}$, where $\alpha \in V \Gamma$ and $\delta \in V \Sigma$.

Proof. By assumption, $K \triangleleft X$ is semiregular on $V \Gamma$ and $X / K \leq$ Aut $\Sigma$ is transitive on $V \Sigma$, so the vertex stabilizers of $X / K$ on $V \Sigma$ are conjugate. Thus, without loss of generality, we may assume $\delta=\alpha^{K} \in V \Sigma$. Then $X_{\alpha} \cong X_{\alpha} K / K \leq(X / K)_{\delta}$. On the other hand, as

$$
\left|X: X_{\alpha}\right|=|V \Gamma|=|K||V \Sigma|=|K|\left|X / K:(X / K)_{\delta}\right| \text {, }
$$

we have $\left|X_{\alpha}\right|=\left|(X / K)_{\delta}\right|$. Hence $X_{\alpha} \cong(X / K)_{\delta}$.

\subsection{Examples}

Clearly, the complete bipartite graph deleting one factor $\mathrm{K}_{n, n}-n \mathrm{~K}_{2}$ is a $\mathbb{Z}_{2}$ cover of $\mathrm{K}_{n}$.

The next family of examples is a subfamily of the graphs $X_{1}(4, q)$, which is first obtained in [7], arising from voltage graphs.

Let $\Sigma$ be a graph and $K$ a finite group. A voltage assignment (or $K$-voltage assignment) of the graph $\Sigma$ is a function $f: A \Sigma \rightarrow K$ such that $f(\alpha, \beta)=f(\beta, \alpha)^{-1}$ for each $(\alpha, \beta) \in A \Sigma$. Then the derived voltage graph $\Sigma \times{ }_{f} K$ from the voltage assignment $f$ is defined with vertex set $V \Sigma \times K$ (Cartesian product), and $(\alpha, g)$ is adjacent to $(\beta, h)$ if and only if $(\alpha, \beta) \in A \Sigma$ and $h g^{-1}=f(\alpha, \beta)$.

Example 3.4. ([7]) Let $q \equiv 3(\bmod 4)$ be a prime power and $F_{q}^{*}$ the multiplicative group of the $q$-elements field $F_{q}$. Let $S(q)$ be the set of all squares in $F_{q}^{*}$ and $N(q)$ the set of all non-squares in $F_{q}^{*}$. Let $\Sigma=\mathrm{K}_{q+1}$, with the vertex set being identified with the projective line $P G(1, q)=F_{q} \cup\{\infty\}$.

Define the graph $X_{1}(4, q)$ be the voltage graph $\sum \times_{f} K$, where $K \cong \mathbb{Z}_{4}$ 
and the voltage assignment $f: A \Sigma \rightarrow K$ is given as following:

$$
f(x, y)= \begin{cases}0 & \text { if } \infty \in\{x, y\} \\ 1 & \text { if } y-x \in S(q) \\ 3 & \text { if } y-x \in N(q)\end{cases}
$$

The following two families of examples are arising from coset graphs, giving rise to examples as in part (2) of Theorem 1.1.

Example 3.5. Suppose that $2^{n}-1 \geq 7$ is a prime and $m=2^{k} m^{\prime}$ with $k \leq 1$ and $m^{\prime} \mid 2^{n-1}-1$. Let $K=\langle a\rangle \cong \mathbb{Z}_{m}, T=\operatorname{PSL}\left(2,2^{n}-1\right)$ and $G=K \times T$, and let $\langle b\rangle:\langle c\rangle \cong \mathbb{Z}_{2^{n}-1}: \mathbb{Z}_{2^{n-1}-1}$ be a maximal parabolic subgroup of $T$. Then the normalizer $N_{T}(\langle c\rangle) \cong D_{2^{n}-2}$. Let $g \in N_{T}(\langle c\rangle)$ be an involution.

Set

$$
\left\{\begin{array}{l}
H^{1}=\left\langle(1, b),\left(a^{2^{k}}, c\right)\right\rangle, \\
\mathcal{G}^{\{1\}}=\operatorname{Cos}\left(G, H^{1}, H^{1}\left(a^{m^{\prime}}, g\right) H^{1}\right) .
\end{array}\right.
$$

Lemma 3.6. Using notations as in Example 3.5. Then $\mathcal{G}^{\{1\}}$ is a G-arc-transitive $\mathbb{Z}_{m}$-cover of $\mathrm{K}_{2^{n}}$.

Proof. Since $\langle b, c\rangle$ is maximal in $T$ and $g \notin\langle b, c\rangle,\langle b, c, g\rangle=T$. Let $X=\left\langle H^{1},\left(a^{m^{\prime}}, g\right)\right\rangle$. Since $T$ is nonabelian simple, we have

$$
\begin{aligned}
X^{\prime} & =\left\langle\left[(1, b),\left(a^{m^{\prime}}, g\right)\right]^{x},\left[\left(a^{2^{k}}, c\right),\left(a^{m^{\prime}}, g\right)\right]^{x} \mid x \in X\right\rangle \\
& \supseteq\left\langle\left(1,[b, g]^{t}\right),\left(1,[c, g]^{t}\right) \mid t \in T\right\rangle=\{1\} \times T^{\prime}=\{1\} \times T,
\end{aligned}
$$

where $X^{\prime}$ and $T^{\prime}$ denote the commutator subgroups of $X$ and $T$, respectively. In particular, $\left(1, g^{-1}\right)$ and $\left(1, c^{-1}\right)$ are in $X$, so $\left(a^{m^{\prime}}, 1\right)$ and $\left(a^{2^{k}}, 1\right)$ are in $X$. It then easily follows $X=G$, so $\mathcal{G}^{\{1\}}$ is connected. Also, as $H^{1} \cong \mathbb{Z}_{2^{n}-1}: \mathbb{Z}_{2^{n-1}-1}$, $\left|V \mathcal{G}^{\{1\}}\right|=\left|G: H^{1}\right|=2^{n} m$, and as $g \in N_{T}(\langle c\rangle), H^{1} \cap\left(H^{1}\right)^{\left(a^{m^{\prime}}, g\right)} \cong \mathbb{Z}_{2^{n-1}-1}$. By Lemma 2.5, $\mathcal{G}^{\{1\}}$ is a $G$-arc-transitive graph of order $2^{n} m$ and primes valency $2^{n}-1$.

Set $N=K \times\{1\}$. Clearly $\mathbb{Z}_{m} \cong N \triangleleft G$. Noting that each element $x \in H^{1}$ has a form $x=\left(a^{2^{k} i}, b^{j} c^{i}\right)$. If $x \in N$, then $b^{j} c^{i}=1$, so $b^{j}=c^{i}=1$, implying $2^{n-1}-1 \mid i$ and $a^{2^{k} i}=1$. Hence $x=1$, that is, $N \cap H^{1}=1$. Now, $N$ is semiregular and has $2^{n}$ orbits on $V \mathcal{G}^{\{1\}}$. By Theorem 2.4, $\mathcal{G}^{\{1\}}$ is a $\mathbb{Z}_{m}$-cover of $\mathcal{G}_{N}^{\{1\}}$. Since $\mathcal{G}_{N}^{\{1\}}$ is of order $2^{n}$ and valency $2^{n}-1, \mathcal{G}_{N}^{\{1\}} \cong \mathrm{K}_{2^{n}}$.

Example 3.7. Suppose that $2^{n}-1 \geq 7$ is a prime and $m=2^{k} m^{\prime}$, where $1 \leq k \leq 2$ and $m^{\prime} \mid 2^{n-1}-1$. Let $K=\langle a\rangle \cong \mathbb{Z}_{m}, \quad S=\operatorname{SL}\left(2,2^{n}-1\right)$ and $G=K \circ S$ is a central product of $K$ and $S$ such that $K \cap S \cong \mathbb{Z}_{2}$. Then there exist $b, c \in S$ and an involution $g \in N_{S}(\langle c\rangle)$ such that $\langle b\rangle:\langle c\rangle \cong \mathbb{Z}_{2^{n}-1}: \mathbb{Z}_{2^{n-1}-1}$, and $\langle b, c, g\rangle=S$.

Set

$$
\left\{\begin{array}{l}
H^{2}=\left\langle b, a^{2^{k}} c\right\rangle, \\
\mathcal{G}^{\{2\}}=\operatorname{Cos}\left(G, H^{2}, H^{2}\left(a^{m^{\prime}} g\right) H^{2}\right) .
\end{array}\right.
$$


Lemma 3.8. Using notations as in Example 3.7. Then $\mathcal{G}^{\{2\}}$ is a $G$-arc-transitive $\mathbb{Z}_{m}$-cover of $\mathrm{K}_{2^{n}}$.

Proof. With similar discussion as in the proof of Lemma 3.6, one may show that $K \cap H=1$ and $\mathcal{G}^{\{2\}}$ is a $G$-arc-transitive graph with order $2^{n} m$ and prime valency $2^{n}-1$. Since $K \triangleleft G$ has $2^{n}$ orbits on $V \Gamma$, by Theorem $2.4, \Gamma$ is an arc-transitive $\mathbb{Z}_{m}$-cover of $\Gamma_{K}$. Since $\Gamma_{K}$ is of order $2^{n}$ and valency $2^{n}-1$, $\Gamma_{K} \cong \mathrm{K}_{2^{n}}$.

We remark that, in Lemmas 3.6 and 3.8, the groups isomorphic to $\mathbb{Z}_{2^{n}-1}: \mathbb{Z}_{2^{n-1}-1}$ are conjugate in $\operatorname{PSL}\left(2,2^{n}-1\right)$ and $\operatorname{SL}\left(2,2^{n}-1\right)$, it is then easy to see that the graphs $\mathcal{G}^{\{1\}}$ and $\mathcal{G}^{\{2\}}$ are independent (up to isomorphism) of the choices of $a, b$.

\section{Proofs of Theorems 1.1 and 1.2}

In this section, we will prove Theorems 1.1 and 1.2.

Proof of Theorem 1.1. Let $X$ be the fibre-preserving group and $K$ the covering transformation group. Then $X$ acts edge-transitively on $\Gamma, K:=\langle a\rangle \cong \mathbb{Z}_{m}$ is normal in $X$, and $Y:=X / K$ acts edge-transitively on $\Sigma$.

If $Y$ is 3-transitive on $V \Sigma$, then $\Sigma$ is $(Y, 2)$-arc-transitive, and so $\Gamma$ is a 2-arc-transitive $\mathbb{Z}_{m}$-cover of $\mathrm{K}_{p^{n}}$. By ([17]: Theorem 1.1), either $m=2$ and $\Gamma \cong \mathrm{K}_{p^{n}, p^{n}}-p^{n} \mathrm{~K}_{2}$, as in part (1)(i) of Theorem 1.1 ; or $m=4$ and $\Gamma \cong X_{1}(4, q)$ with $q=p^{n}-1 \equiv 3(\bmod 4)$, so $p=2$, and by Lemma 3.1 , $q=2^{n}-1 \geq 7$ is a prime, as in part (1)(ii) of Theorem 1.1 .

Thus, from now on, suppose that $Y$ is not 3-transitive on $V \Gamma$. Since $Y$ acts edge-transitively on $\mathrm{K}_{p^{n}}, Y$ is 2-homogeneous (so primitive) on $V \Gamma$ of degree $p^{n}$. Hence, either $Y$ is affine or almost simple. Let $\alpha \in V \Gamma$ and $\delta \in V \Sigma$.

Suppose first $Y$ is almost simple with socle $T$. Then $T$ is transitive on $V \Sigma$, so $\left|T: T_{\delta}\right|=p^{n}$. Hence the tuple $\left(T, T_{\delta}\right)$ (as $(T, H)$ there) satisfies Proposition 2.1. Noting that, if $T$ satisfies parts (i, iv, v), then $T$ is $s$-transitive with $s \geq 3$, which is not the case; if $T=\operatorname{PSU}(4,2)$ as in part (vi), then $Y \leq \operatorname{Aut}(T)=\operatorname{PSU}(4,2) \cdot \mathbb{Z}_{2}$ is not 2-homogeneous, also not the case. So $\left(T, T_{\delta}\right)$ satisfies parts (ii) and (iii) of Proposition 2.1. In both cases, $T$ is 2-transitive on $V \Sigma$ and so is arc-transitive on $\Sigma$, hence $G:=K . T \triangleleft X$ is arc-transitive on $\Gamma$. By Lemma 2.6, $G=K . T$ is a central extension, and by Lemma 3.3, $G_{\alpha} \cong T_{\delta}$.

Assume $\left(T, T_{\delta}\right)=\left(\operatorname{PSL}(2,11), \mathrm{A}_{5}\right)$. Then $\Sigma=\mathrm{K}_{11}, G=K . \operatorname{PSL}(2,11)$ and $G_{\alpha} \cong \mathrm{A}_{5}$. As the Schur multiplier $\operatorname{Mult}(\operatorname{PSL}(2,11)) \cong \mathbb{Z}_{2}$, by Lemma 2.6, $G^{\prime} \cong \operatorname{PSL}(2,11)$, or $\operatorname{SL}(2,11)$ with $m$ even. Since $G^{\prime} \triangleleft G, G_{\alpha}^{\prime} \triangleleft G_{\alpha} \cong \mathrm{A}_{5}$, implying $G_{\alpha}^{\prime}=1$ or $\mathrm{A}_{5}$. If $G_{\alpha}^{\prime}=1$, Lemma 3.3 implies $\mathrm{A}_{5} \cong G_{\alpha} \leq K /\left(K \cap G^{\prime}\right)$, which is a contradiction. So $G_{\alpha}^{\prime} \cong \mathrm{A}_{5}$, and hence $G^{\prime} \nsubseteq \operatorname{SL}(2,11)$ as $\operatorname{SL}(2, r)$ with $r \geq 5$ an odd prime has a unique involution by ([18]: Lemma 7.4). Thus $G^{\prime} \cong \operatorname{PSL}(2,11), G=K \times G^{\prime}$, and $G^{\prime}$ has exactly $|K|=m$ orbits on $V \Gamma$. If $m \geq 3$, then $\Gamma_{G^{\prime}}$ is $G / M$-arc-transitive of order $|K|$, where $M \supset G^{\prime}$ is the kernel of $G$ acting on the set of $G^{\prime}$-orbits on $V \Gamma$, which is a contradiction as $G / M \cong K /(K \cap M)$ is cyclic. Thus $m=2$ and $G^{\prime}$ has exactly two orbits on $V \Gamma$, by ([17]: Lemma 2.4), $\Gamma \cong \mathrm{K}_{11,11}-11 \mathrm{~K}_{2}$ is the standard double cover of 
$\Sigma=\mathrm{K}_{11}$, as in part (1)(i) of Theorem 1.1.

Assume now $T=\operatorname{PSL}(d, q)$ with $p^{n}=\frac{q^{d}-1}{q-1}$, as in part (ii) of Proposition

2.1. By Lemma 3.1, $d$ is a prime. We divided our discussion into three cases.

Case (i). Suppose $\operatorname{Mult}(T) \cong \mathbb{Z}_{(d, q-1)}$ and $d \mid q-1$.

In this case, $p^{n}=1+q+\cdots+q^{d-1} \equiv 0(\bmod d)$, so $d=p$ and $q=1+p t$ for some $t \geq 1$. It follows

$$
\begin{aligned}
p^{n+1} t & =(1+p t)^{p}-1 \\
& =1+p^{2} t+\frac{p(p-1)}{2}(p t)^{2}+\cdots+(p t)^{p}-1 \\
& =p^{2} t\left(1+\frac{p(p-1)}{2} t+\cdots+p^{p-2} t^{p-1}\right),
\end{aligned}
$$

so $p^{n-1}=1+\frac{p(p-1)}{2} t+\cdots+p^{p-2} t^{p-1}$, implying $p=2$. Now, $2^{n}-1=q$ is a prime power, by Lemma 3.1, we further have that $q \geq 7$ is a prime. Hence, $T=\operatorname{PSL}\left(2,2^{n}-1\right), \quad \Sigma=\mathrm{K}_{2^{n}}$ is of prime valency $2^{n}-1$, and so is $G$-locallyprimitive. As $G=K . T$ is a central extension and $\operatorname{Mult}(T) \cong \mathbb{Z}_{2}$, by Lemma 2.6, $G^{\prime} \cong \operatorname{PSL}\left(2,2^{n}-1\right)$ or $\operatorname{SL}\left(2,2^{n}-1\right)$. Since $G_{\alpha}^{\prime} \triangleleft G_{\alpha} \cong T_{\delta} \cong \mathbb{Z}_{2^{n}-1}: \mathbb{Z}_{2^{n-1}-1}$, a parabolic maximal subgroup of $T$, either $G_{\alpha}^{\prime}=1$ or $G_{\alpha}^{\prime} \cong \mathbb{Z}_{2^{n}-1}: \mathbb{Z}_{l}$ for some $l \mid 2^{n-1}-1$. If $G_{\alpha}^{\prime}=1$, Lemma 3.2 implies that $G_{\alpha} \leq K /\left(K \cap G^{\prime}\right)$ is cyclic, which is contradicts. Thus $G_{\alpha}^{\prime} \cong \mathbb{Z}_{2^{n}-1}: \mathbb{Z}_{l}$.

Assume $G^{\prime} \cong \operatorname{PSL}\left(2,2^{n}-1\right)$. Then $G=K \times G^{\prime}$. Since $|V \Gamma|=2^{n} m$ and $\left|\alpha^{G^{\prime}}\right|=\frac{\left|\operatorname{PSL}\left(2,2^{n}-1\right)\right|}{\left(2^{n}-1\right) l}=\frac{2^{n}\left(2^{n-1}-1\right)}{l}, G^{\prime}$ has $\frac{m l}{2^{n-1}-1}$ orbits on $V \Gamma$. If $\frac{m l}{2^{n-1}-1} \geq 3$, by Theorem 2.4, $\Gamma_{G^{\prime}}$ is $G / G^{\prime}$-locally-primitive of valency $2^{n}-1$, which is a contradiction as $G / G^{\prime}$ is abelian. Thus $\frac{m l}{2^{n-1}-1} \leq 2$ and $m \mid 2^{n}-2$. Write $m=2^{k} m^{\prime}$, where $0 \leq k \leq 1$ and $m^{\prime} \mid 2^{n-1}-1$. Since $\left(m, 2^{n}-1\right)=1$, each element of order $2^{n}-1$ of $G$ has a form $(1, b)$ with $b \in G^{\prime}$, and each cyclic subgroup of order $2^{n-1}-1$ of $G$ has a form $\left\langle\left(a^{2^{k}}, c\right)\right\rangle$ with $i \mid m^{\prime}$ and $c \in G^{\prime}$, we thus may suppose $G_{\alpha}=\langle(1, b)\rangle:\left\langle\left(a^{2^{k_{i}}}, c\right)\right\rangle$. As $\left(a^{a^{k_{i}}}, c\right)^{o(c)}=\left(a^{2^{k_{i}} o(c)}, 1\right) \in K \cap G_{\alpha}=1$, we have $o\left(a^{2^{k_{i}}}\right) \mid o(c)$ and hence $o(c)=o\left(a^{2^{k_{i}}}, c\right)=2^{n-1}-1$. Also, noting that a 2-element of $G$ can be expressed as $\left(a^{m^{\prime} j}, g\right)$, where $0 \leq j \leq 1$ and $g \in G^{\prime}$ is a 2-element. By Lemma 2.5, we may suppose $\Gamma \cong \operatorname{Cos}\left(G, G_{\alpha}, G_{\alpha}\left(a^{m^{\prime} j}, g\right) G_{\alpha}\right)$. Since $\operatorname{val}(\Gamma)=2^{n}-1, g \in N_{T}(\langle c\rangle)$. By the connectedness of $\Gamma,\left\langle G_{\alpha},\left(a^{m^{\prime} j}, g\right)\right\rangle=G$, it follows that $\langle b, c, g\rangle=G^{\prime}$ and $\left\langle a^{2^{k_{i}}}, a^{m^{\prime j}}\right\rangle=\langle a\rangle$, implying $i=j=1$ as $i \mid 2^{n-1}-1$ and $0 \leq j \leq 1$. Hence $\Gamma \cong \mathcal{G}^{11\}}$ as in Example 3.5, and which is really an example by Lemma 3.6.

Assume now $G^{\prime} \cong \operatorname{SL}\left(2,2^{n}-1\right)$. Then $m$ is even and $G=K \circ G^{\prime}$ is a central product with $K \cap G^{\prime} \cong \mathbb{Z}_{2}$. Because $\left|\alpha^{G^{\prime}}\right|=\frac{\left|\operatorname{SL}\left(2,2^{n}-1\right)\right|}{\left(2^{n}-1\right) l}=\frac{2^{n+1}\left(2^{n-1}-1\right)}{l}, G^{\prime}$ 
has $\frac{m l}{2^{n}-2}$ orbits on $V \Gamma$. If $\frac{m l}{2^{n}-2} \geq 3$, Theorem 2.4 implies that $\Gamma_{G^{\prime}}$ is $G / G^{\prime}$ -locally-primitive, which is impossible because $G / G^{\prime}$ is abelian. So $\frac{m l}{2^{n}-2} \leq 2$ and $m \mid 2^{n+1}-4$. Set $m=2^{r} m^{\prime}$, where $1 \leq r \leq 2$ and $m^{\prime} \mid 2^{n-1}-1$. As each 2element of $G$ can be expressed as $a^{m^{\prime} j} g$, where $0 \leq j \leq 2$, and $g \in G^{\prime}$ is a 2element. By Lemma 2.5, we may assume $\Gamma \cong \operatorname{Cos}\left(G, G_{\alpha}, G_{\alpha}\left(a^{m^{\prime} j} g\right) G_{\alpha}\right)$. Now, with similar discussion as in the above paragraph, one may prove that $j=1$ and $\Gamma \cong \mathcal{G}^{\{2\}}$ as in Example 3.7, giving rise to example by Lemma 3.8.

Case (ii). Suppose $\operatorname{Mult}(T) \cong \mathbb{Z}_{(d, q-1)}$ and $d \nmid q-1$.

Recall that $d$ is a prime, $\operatorname{Mult}(T)=1$. It then follows from Lemma 2.6 that $G^{\prime} \cong T$, and $G=K \times G^{\prime} \cong \mathbb{Z}_{m} \times \operatorname{PSL}(d, q)$.

If $d=2$, as $d \nmid q-1, q$ is 2 -power, so $Y \geq T=\operatorname{PSL}(2, q)=\operatorname{PGL}(2, q)$ is 3transitive on $V \Sigma$, which contradicts the assumption before.

Thus $d \geq 3$, and

$$
G_{\alpha} \cong T_{\delta} \cong\left[q^{d-1}\right] \cdot \mathbb{Z}_{q-1} \cdot \operatorname{PSL}(d-1, q) \cdot \mathbb{Z}_{(d-1, q-1)},
$$

a maximal parabolic subgroup of $T$, where $\left[q^{d-1}\right]$ denotes the elementary abelian group of order $q^{d-1}$. If $G^{\prime}$ has at least three orbits on $V \Gamma$, then $\Gamma_{G^{\prime}}$ is $G / P$-arc-transitive, where $P$ is the kernel of $G$ acting on the $G^{\prime}$-orbits in $V \Gamma$, which is not possible as $P \geq G^{\prime}$ and $G / G^{\prime} \cong K$ is cyclic. So $G^{\prime}$ has at most two orbits on $V \Gamma$. By Lemma 3.2, $G_{\alpha} / G_{\alpha}^{\prime} \leq K /\left(K \cap G^{\prime}\right)=K$ is cyclic, and as $\left|G: G_{\alpha}\right|=\left|G^{\prime}: G_{\alpha}^{\prime}\right|$ or $2\left|G: G_{\alpha}^{\prime}\right|,\left|G_{\alpha}: G_{\alpha}^{\prime}\right|=m$ or $m / 2$, we conclude that $m \mid 2(d-1, q-1)$, as in part (3) of Theorem 1.1 .

Case (iii). Suppose $\operatorname{Mult}(T) \nRightarrow \mathbb{Z}_{(d, q-1)}$.

Since $\frac{q^{d}-1}{q-1}=p^{n}$ is a prime power, checking the candidates in Lemma 2.7, the only possibility is $\operatorname{Mult}(T) \cong \mathbb{Z}_{2}$, and either $(d, q)=(2,4)$ and $|V \Sigma|=5$, or $(d, q)=(3,2)$, and $|V \Sigma|=7$. For the former case, $T \cong \operatorname{PSL}(2,4) \cong \mathrm{A}_{5}$ is 3 transitive on $V \Sigma$, so is $Y$, which contradicts the former assumption.

Consider the latter case. Then $T \cong \operatorname{PSL}(3,2) \cong \operatorname{PSL}(2,7), G_{\alpha} \cong T_{\delta} \cong \mathrm{S}_{4}$ and $G^{\prime} \cong \operatorname{PSL}(2,7)$ or $\operatorname{SL}(2,7)$ by Lemma 2.6. By Lemma 3.2, we have $G_{\alpha}^{\prime} \neq 1$, so $G_{\alpha}^{\prime} \cong \mathbb{Z}_{2}^{2}, \mathrm{~A}_{4}$ or $\mathrm{S}_{4}$ and hence $G^{\prime} ¥ \mathrm{SL}(2,7)$ by ([18]: Lemma 7.4 ). Thus $G^{\prime} \cong \operatorname{PSL}(2,7)$ and $G=K \times G^{\prime}$. Since $G / G^{\prime}$ is abelian, $G^{\prime}$ has at most two orbits on $V \Gamma$ by Theorem 2.4. If $G^{\prime}$ is transitive on $V \Gamma$, then $\mathrm{S}_{4} \cong G_{\alpha}=G_{\alpha}^{\prime} \cdot \mathbb{Z}_{m}$, so $m=2$ and $G_{\alpha}^{\prime}=\mathrm{A}_{4}$, a simple computation by using Magma [19] shows that no example exists in this case. If $G^{\prime}$ has 2 orbits on $V \Gamma$, then $G_{\alpha}=G_{\alpha}^{\prime} \cdot \mathbb{Z}_{m}$ with $m$ even, it follows that either $m=2$ and $G_{\alpha}^{\prime}=G_{\alpha}=\mathrm{S}_{4}$, or $m \stackrel{\overline{2}}{=} 4$ and $G_{\alpha}^{\prime}=\mathrm{A}_{4}$. For the former case, by ([17]: Lemma 2.4), $\Gamma=\mathrm{K}_{7,7}-7 \mathrm{~K}_{2}$. For the latter case, no example occurs by Magma [19].

Suppose now that $Y$ is affine. Then $T=\mathbb{Z}_{p}^{n}$, and $\Sigma$ is a $Y$-normal edgetransitive Cayley graph of $T$, and in turn, $\Gamma$ is an $X$-normal edge-transitive Cayley graph of K.T. This completes the proof of Theorem 1.1.

Proof of Theorem 1.2. Let $X$ be the fibre-preserving group and let 
$K:=\langle a\rangle \cong \mathbb{Z}_{m}$ be the covering transformation group. Then $K \triangleleft X, Y:=X / K$ acts locally-primitively on $\Gamma$ and hence satisfies Theorem 1.1. If $\Gamma$ satisfies part (3) of Theorem 1.1, then $Y \leq \operatorname{Aut}(\operatorname{PSL}(d, q))$ with $d \geq 3$ acts locallyprimitively on $\Sigma$, so $Y$ is 2-primitive on the set of project points, which is a contradiction by ([20]: Lemma 2.5).

Assume that $\Gamma$ satisfies part (4) of Theorem 1.1. Then $Y$ is affine with socle $T \cong \mathbb{Z}_{p}^{n}$, and $\Sigma$ is a $Y$-normal locally-primitive Cayley graph of $T$. By Lemma 2.3, $p=2$. Let $C=C_{X}(K)$. Then $C \geq K$. If $C=K$, then $Y=X / K \leq \operatorname{Aut}\left(\mathbb{Z}_{m}\right)$ is abelian, which is a contradiction.

Thus, $C>K$. Then $1 \neq C / K \triangleleft Y$, and so $T \triangleleft C / K$ as $T$ is the unique minimal normal subgroup of $Y$. Let $R \triangleleft X$ such that $R \subseteq C$ and $R / K \cong T$. Then $R \cong K . T$ is a central extension, and $\Gamma$ is an $X$-normal locally-primitive Cayley graph of $R$. Write $m=2^{d} m^{\prime}$ with $m^{\prime}$ odd. If $m^{\prime}>1$, then $N:=\left\langle a^{2^{d}}\right\rangle \triangleleft X$, and by Theorem 2.4, $\Gamma_{N}$ is an $X / N$-normal locally-primitive Cayley graph of $R / N$. However, as $R / N \cong \mathbb{Z}_{m^{\prime}} \cdot \mathbb{Z}_{2}^{n}$ is a central extension, we have $R / N \cong \mathbb{Z}_{m^{\prime}}: \mathbb{Z}_{2}^{n}$ is abelian, by Lemma 2.3, which is a contradiction. Hence $m=2^{d}$, and $\Gamma$ satisfies part (4) of Theorem 1.2.

Corollary 1.3 follows immediately by Theorem 1.2 . We finally prove Corollary 1.4 .

By assumption, $\Gamma$ satisfies part (1)(i) or (2)(iii) of Theorem 1.2 with $n=2$. For the former, part (1) of Corollary 1.4 holds. For the latter, $\Sigma \cong K_{4}$, it then follows from ([4]: Theorem 6.1) that either $\Gamma \cong \mathrm{K}_{4,4}-4 \mathrm{~K}_{2}$, or $\Gamma \cong P(8,3)$ is the generalized Peterson graph as in Corollary 1.4(2).

\section{Conclusion}

In this paper, a characterization of edge-transitive cyclic covers of complete graphs with prime power order is given by using the techniques of finite group theory and the related properties of coset graphs. Certain previous results are generalized and some new families of examples are founded.

\section{Supported}

This work was partially supported by National Natural Science Foundation of China (11801252); Doctor Program of Yunnan Normal University (No. 2015ZB002).

\section{Conflicts of Interest}

The authors declare no conflicts of interest regarding the publication of this paper.

\section{References}

[1] Praeger, C.E. (1992) An O'nan-Scott Theorem for Finite Quasiprimitive Permutation Groups and an Application to 2-Arc Transitive Graphs. Journal of the London Mathematical Society, 47, 227-239. https://doi.org/10.1112/jlms/s2-47.2.227

[2] Conder, M. and Ma, J.C. (2013) Arc-Transitive Abelian Regular Covers of Cubic Graphs. Journal of Algebra, 387, 215-242. 
https://doi.org/10.1016/j.jalgebra.2013.02.035

[3] Conder, M. and Ma, J.C. (2013) Arc-Transitive Abelian Regular Covers of the Heawood Graph. Journal of Algebra, 387, 243-267.

https://doi.org/10.1016/j.jalgebra.2013.01.041

[4] Feng, Y.Q. and Kwak, J.H. (2007) Cubic Symmetric Graphs of Order A Small Number Times a Prime or a Prime Square. Journal of Combinatorial Theory, Series $B, 97,627-646$. https://doi.org/10.1016/j.jctb.2006.11.001

[5] Malnič, A., Marušič, D. and Potočnik, P. (2004) Elementary Abelian Covers of Graphs. Journal of Algebraic Combinatorics, 20, 71-97. https://doi.org/10.1023/B:JACO.0000047294.42633.25

[6] Pan, J.M., Huang, Z.H. and Xu, F.H. (2014) On Cyclic Regular Covers of Complete Graphs of Small Order. Discrete Mathematics, 331, 36-42. https://doi.org/10.1016/j.disc.2014.04.023

[7] Du, S.F., Marušič, D. and Waller, A.O. (1998) On 2-Arc-Transitive Covers of Complete Graphs. Journal of Combinatorial Theory, Series B, 74, 276-290.

https://doi.org/10.1006/jctb.1998.1847

[8] Du, S.F., Kwak, J.H. and Xu, M.Y. (2005) On 2-Arc-Transitive Covers of Complete Graphs with Covering Transformation Group $\mathbb{Z}_{p}^{3}$. Journal of Combinatorial Theory, Series B, 93, 73-93. https://doi.org/10.1016/j.jctb.2003.09.003

[9] Robinson, D.J.S. (1982) A Course in the Theory of Groups. Springer-Verlag, New York. https://doi.org/10.1007/978-1-4684-0128-8

[10] Li, C.H. (2006) Finite Edge-Transitive Cayley Graphs and Rotary Cayley Maps. Transactions of the American Mathematical Society, 358, 4605-4635. https://doi.org/10.1090/S0002-9947-06-03900-6

[11] Guralnick, R.M. (1983) Subgroups of Prime Power Index in a Simple Group. Journal of Algebra, 225, 304-311. https://doi.org/10.1016/0021-8693(83)90190-4

[12] Huppert, B. (1967) Endliche Gruppen I. Springer-Verlag, Berlin. https://doi.org/10.1007/978-3-642-64981-3

[13] Li, C.H. and Pan, J.M. (2007) Finite 2-Arc-Transitive Abelian Cayley Graphs. European Journal of Combinatorics, 29, 148-158. https://doi.org/10.1016/j.ejc.2006.12.001

[14] Lorimer, P. (1984) Vertex-Transitive Graphs: Symmetric Graphs of Prime Valency. Journal of Graph Theory, 8, 55-66. https://doi.org/10.1002/jgt.3190080107

[15] Sabidussi, G.O. (1984) Vertex-Transitive Graphs. Monatshefte für Mathematik, 68, 426-438. https://doi.org/10.1007/BF01304186

[16] Gorenstein, D. (1982) Finite Simple Groups. Plenum Press, New York. https://doi.org/10.1007/978-1-4684-8497-7

[17] Li, C.H., Lou, B.G. and Pan, J.M. (2010) Finite Locally Primitive Abelian Cayley Graphs. Science China, 53, 1-10. https://doi.org/10.1007/s11430-010-4140-7

[18] Isaacs, I.M. (2008) Finite Group Theory. American Mathematics Society, Providence. https://doi.org/10.1090/gsm/092

[19] Bosma, W., Cannon, J. and Playoust, C. (1997) The Magma Algebra System I: The User Languages. Journal of Symbolic Computation, 24, 235-265. https://doi.org/10.1006/jsco.1996.0125

[20] Li, C.H., Pan, J.M. and Ma, L. (2009) Locally Primitive Graphs of Prime-Power Order. Journal of the Australian Mathematical Society, 86, 111-122. https://doi.org/10.1017/S144678870800089X 